\title{
Implicit definability of truth constants in Łukasiewicz logic
}

\author{
Zuzana Haniková \\ Institute of Computer Science \\ Czech Academy of Sciences \\ 18207 Prague, Czech Republic \\ hanikova@cs.cas.cz
}

October 16, 2018

\begin{abstract}
In the framework of propositional Łukasiewicz logic, a suitable notion of implicit definability, tailored to the intended real-valued semantics and referring to the elements of its domain, is introduced. Several variants of implicitly defining each of the rational elements in the standard semantics are explored, and based on that, a faithful interpretation of theories in Rational Pavelka logic in theories in Łukasiewicz logic is obtained. Some of these results were already presented in [12] as technical statements. A connection to the lack of (deductive) Beth property in Łukasiewicz logic is drawn. Moreover, while irrational elements of the standard semantics are not implicitly definable by finitary means, a parallel development is possible for them in the infinitary Łukasiewicz logic. As an application of definability of the rationals, it is shown how computational complexity results for Rational Pavelka logic can be obtained from analogous results for Lukasiewicz logic. The complexity of the definability notion itself is studied as well. Finally, we review the import of these results for the precision/vagueness discussion for fuzzy logic, and for the general standing of truth constants in Łukasiewicz logic.
\end{abstract}

\section{Introduction}

This paper is a contribution to the study of truth constants in Łukasiewicz logic. Previous works on the subject, often in a wider context of other t-norm based logics, include [22, 12, 14, 8, 5]. Predominantly, this paper is about constants for rational elements of [0,1], but an analogous development for irrational constants in the infinitary variant of Eukasiewicz logic is suggested. Since truth constants are native to propositional logic, the entire paper stays on the propositional level.

Two logics are juxtaposed in this paper: (infinite-valued) Eukasiewicz logic $\mathrm{E}$ and Rational Pavelka logic RPL. The former was introduced by Jan Łukasiewicz, with later simplifications by himself and others (see [17]). The latter, RPL, was introduced by Hájek in [12] as a considerable simplification of earlier systems for reasoning with partially true statements as envisaged by Goguen [11] and proposed by Pavelka 22]. Many resources discuss one or both of these logics very accurately, but two excellent sources are [12] and [14].

In Section 3, we start with providing a semantic notion of implicit definability, referring to the standard MV-algebra $[0,1]_{\mathrm{E}}$. This is in accord with the ontology of truth constants (rational or other) in Łukasiewicz logic, which arise from the real-valued semantics (intended, 
and presumably therefore called "standard") and permeate the syntax. Using the fact that that each rational is definable in $[0,1]_{\mathrm{E}}$ under this semantic definability notion, we faithfully interpret RPL in a particular theory over $\mathrm{E}$; this interpretation result was first presented in [12], where a different evidence for implicit definability is provided. Moreover, [14, Theorem 2.6] offers what is presumably the next best thing to rational constants being term definable: for each formula $\varphi$ of RPL and positive integer $n$ divisible by denominators of truth constants in $\varphi$, there is an E-formula $\psi$ s.t. RPL $\vdash \varphi^{n} \equiv \psi$. Along the way, we also discuss other methods of obtaining implicit definability of rationals; e.g., one can rely on the bookkeeping formulas, which do the job very adequately, or one can refer to formulas representing certain McNaughton functions.

Mimicking the finitary definition in (finitary) Eukasiewicz logic, a non-finitary definability notion for irrationals in infinitary Lukasiewicz logic $\mathrm{E}_{\infty}$ is outlined, with some analogous properties (e.g., under a theory containing the defining formulas, valid bookkeeping statements for the irrationals can be derived in the logic).

Implicit definability is evocative of Beth property. We briefly consider this (somewhat broader) context, and discuss how our technique provides another way of demonstrating the lack of (deductive) Beth property in Eukasiewicz logic, proved in [16]. The status of RPL with respect to Beth property seems to be unknown.

We then weigh the message of this interpretability result, and exploit it somewhat. It seems to be often overlooked that a considerable part of the metamathematics of Lukasiewicz logic with constants is already addressed by (a special case of) the metamathematics of theories over Łukasiewicz logic. As an example of this phenomenon and an application of the interpretation result, in Section 4 we inspect the result on complexity of propositional $\mathrm{RPL}$, that is, the recognition of theoremhood and finite consequence relation. Both are coNPcomplete; we show that this result is implied by (the same) complexity results for $\mathrm{E}$. We also show that the notion of definability as such, taken as a decision problem, is hard.

Finally, apparently the defining formulas allow for speaking about arbitrary rational truth values, which brings to mind previous discussions of the artificial precision problem in fuzzy logic. We briefly touch this topic in Section 6 .

\section{Preliminaries}

This paper deals with propositional Łukasiewicz logic and its algebraic semantics. It makes no distinction between propositional formulas (in the language of Łukasiewicz or other logics, possibly expanding the language) and terms of the language of the algebraic semantics; thus formulas and terms are the same objects. Similarly, we conflate propositional connectives with function symbols.

Although Łukasiewicz logic can be presented in several very succinct sets of basic connectives, we make use of a variety of connectives: the language of $\mathrm{FL}_{\mathrm{ew}}$, namely, the set $\{\cdot, \rightarrow, \wedge, \vee, \overline{0}, \overline{1}\}$, the negation $\neg$, the strong disjunction $\oplus$, and the equivalence $\equiv$. The expression $x^{n}$ stands for $\underbrace{x \cdot x \cdot \ldots x}_{n \text { times }}$ and $n x$ stands for $\underbrace{x \oplus x \oplus \ldots x}_{n \text { times }}$.

Łukasiewicz logic is amply presented and discussed in literature (see [4, 7, 21]); this paper takes for granted the reader's familiarity with the basics. A general semantics of Eukasiewicz logic is given by the variety of MV-algebras. The standard $\mathrm{MV}$-algebra $[0,1]_{\mathrm{E}}$ is given by the Łukasiewicz t-norm on the domain of the real unit interval $[0,1]$. While $\mathrm{E}$ is strongly complete 
w.r.t. MV-algebras, it only enjoys finite strong standard completeness, but not strong standard completeness (see [12, 6]). The finite MV-chain with $n+1$ elements is denoted $\mathcal{L}_{n+1}$.

Rational Pavelka logic RPL (see [12]) expands the language of Łukasiewicz logic with constants for rationals in $[0,1]$. Extending the notational convention for the two constants $\overline{0}$ and $\overline{1}$, we use horizontal bars to distinguish the constant $\bar{r}$ from its intended interpretation $r$ (this convention extends also to irrationals used here) 1 The intended interpretation, i.e., $\bar{r}$ being interpreted by $r$ for each $r \in \mathbb{Q}$, is referred to as the canonical interpretation of constants, to distinguish it from other interpretations, given by algebras that contain an isomorphic copy of (a subalgebra of) the standard MV-algebra on the rationals.

A bookkeeping formula (see [12]) is a formula recording the behaviour of an algebraic operation on elements of the domain interpreting the constants, predominantly, the rationals in $[0,1]$; this domain is closed under the operations of $[0,1]_{\mathrm{E}}$. For example, $\overline{6 / 13} \rightarrow \overline{5 / 13} \equiv$ $\overline{12 / 13}$ is a bookkeeping formula. It is quite usual to include all such bookkeeping formulas that are valid under the canonical interpretation in $[0,1]_{\mathrm{L}}$ as axioms of a logic featuring constants; the bookkeeping axioms are then the only new axioms that are added. This is indeed the case of RPL as an axiomatic expansion of $\mathrm{E}$.

We do not consider a general semantics for RPL, only the standard one, whose E-reduct is the algebra $[0,1]_{\mathrm{E}}$. By slight abuse of language, we use the notation $[0,1]_{\mathrm{E}}$ also for the RPL-algebra that expands the standard MV-algebra with a canonical interpretation of each rational constant, or even for an algebra that further expands the language with constants whose intended interpretation is irrational (Subsection 3.4).

RPL enjoys finite strong standard canonical completeness (see [8]): that is, for a finite $T, \varphi$ in the language of RPL, we have $T \vdash_{\mathrm{RPL}} \varphi$ if and only if $T$ entails $\varphi$ in $[0,1]_{\mathrm{L}}$ under the canonical interpretation of constants 2

Finite strong canonical completeness of RPL (and finite strong completeness of $\mathrm{E}$ ) entails a conservativity result for RPL over $\mathrm{E}$ for propositional case; the conservativity proof for the two first-order logics is more laborious (see [14]). Although the proof is based on finite strong standard completeness, we do have that if $T$ is infinite and $T, \varphi$ are without constants, then $T \vdash_{\mathrm{RPL}} \varphi$ if and only if $T \vdash_{\mathrm{L}} \varphi$.

\section{Definability}

A truth constant is specified within the language of a propositional calculus: it is a nullary connective, its behaviour determined by the axioms and rules of the calculus. Providing a semantics for the calculus involves suggesting an interpretation of its terms, including the constants.

Nevertheless, in addition to constants specified by the language, some other terms in the language may behave as constants too. As a ready example, many terms of either classical or fuzzy propositional logic behave as the constants $\overline{0}$ and $\overline{1}$ : we refer to them as contradictions and tautologies respectively. Here "behave as" can have either of two meanings: the constant $\overline{0}$ is in the language anyway and the term in question is provably equivalent to it, or it is not in the language and one relies on the a semantics where the term in question always evaluates

\footnotetext{
${ }^{1}$ We also use a horizontal bar to denote complement of a set. A vector of variables is denoted with a tilde ( such as $\tilde{x}$ ).

${ }^{2}$ In fact, the canonical interpretation of constants is the only one that consistently expands $[0,1]_{\mathrm{E}}$ under the usual bookkeeping axioms; see Section 3
} 
to the intended evaluation of the (possibly absent) constant 3 In either case, one would speak about term definability. We return to this kind of definability in Section 3.3. Recalling that the set of contradictory terms and the set of tautologous terms of classical propositional are both coNP complete, the simple example above also hints that it is nontrivial to determine if a given term behaves as a particular constant.

Definition 3.1. Let $a \in[0,1]$. The value a is term-definable in $[0,1]_{\mathrm{L}}$ if and only if there is an $M V$-term $\varphi$ such that $v(\varphi)=$ a for each valuation $v$ in $[0,1]_{\mathrm{E}}$.

In the standard MV-algebra $[0,1]_{\mathrm{L}}$, no constants beyond the classical ones are termdefinable: constant non-integer functions are not McNaughton functions ([19]). So the decision problem "Does $\varphi$ term-define $x$ ?" is limited to $x \in\{0,1\}$; we are asking about unsatisfiable or tautologous terms in the real-valued semantics of propositional Łukasiewicz logic.

\subsection{Defining the rationals}

This section aims to expose a phenomenon that is one step more nuanced. Namely, some variables may behave as constants in the framework of a particular theory: in each model of the theory, the value of such variables is fixed. We will discuss (propositional) implicit definability. In fact we shall present two different concepts: implicit definability by a term (i.e., a finite theory) and a variant thereof where the defining theory is necessarily infinite and the logic considered is not finitary. The focus of this paper is on the former concept, which employs the more frequently considered finitary Łukasiewicz logic $\mathrm{E}$.

Definition 3.2. Let $\mathcal{A}$ be an $M V$-algebra, a an element of its domain, $\varphi\left(x_{1}, \ldots, x_{n}\right)$ an $M V$ term, and $1 \leq i \leq n$. The term $\varphi$ implicitly defines the element $a$ in variable $x_{i}$ in $\mathcal{A}$ if and only if

- $\varphi$ is satisfiable in $\mathcal{A}$, and

- $v(\varphi)=1^{\mathcal{A}}$ implies $v\left(x_{i}\right)=a$ for each $\mathcal{A}$-valuation $v$.

An element $a \in \mathcal{A}$ is definable in $\mathcal{A}$ if and only if there is an $M V$-term that defines it there.

It is not difficult to come up with a suitable theory that implicitly defines rationals in $[0,1]_{\mathrm{E}}$. Indeed a theory $T$ in variables indexed by the rationals (say, $x_{m / n}$ for $m, n$ coprime), and with just the usual bookkeeping formulas for these variables, obtained from the corresponding MV-operations on the indices of the variables (e.g., $x_{6 / 13} \rightarrow x_{5 / 13} \equiv x_{12 / 13}$ ), as the axioms of $T$, makes all the variables $x_{m / n}$ behave just as the constant $\overline{m / n}$ would behave in RPL. $T$ is infinite, so it does not follow the requirement of implicit definability (of each of the rationals) by a term; however, one quickly observes that to implicitly define $m / n$, only constants with denominators $n$ are needed. This allows for a much narrower theory $T$ that is finite and has a unique interpretation (namely, the canonical one) in $[0,1]_{\mathrm{L}}$. We shall return to this claim at the end of Subsection 3.2 by providing a sketchy example, and we also discuss a redundancy issue there.

\footnotetext{
${ }^{3}$ The discourse in mathematical fuzzy logic so far confirms that fuzzy logics are semantics-based; accordingly, constants are tied to the intended (real-valued) semantics, standing for the rational or the real numbers thereof. Indeed, where all rational constants from the interval $[0,1]$ are present, one may claim that semantics has leaked into syntax in a substantial way, in particular, such a process narrows down the range of algebraic interpretations of the calculus noticeably.
} 
Another way to implicitly define a rational $a$ is to rely on McNaughton functions: there is a plethora of one-variable McNaughton functions that attain the value 1 on the singleton $\{a\}$, each of them corresponds to an MV-term, and each such term implicitly defines $a$.

One construction using yet different term to achieve implicit definability was already given in [12. The book also presents a faithful interpretation of theories in RPL in theories in $\mathrm{E}$, which we reproduce below. We use the various versions of proving implicit definability for a general point to be made in a subsequent section.

We offer a simple variant of the definability axioms. They are only marginally different from bookkeeping, one of the differences being that $1 / n$ is defined by a single term without additional variables.

\section{$3.2 \quad$ A theory of constants}

From now on, definability and implicit definability may be conflated to the latter, for the sake of brevity.

The following technical lemma is a useful tool for MV-algebras (cf. [23, 10, 15]).

Lemma 3.3. Let $\mathcal{A}$ be an $M V$-chain and $n \geq 2$ an integer. The equation $x=(\neg x)^{n-1}$ has a solution in $\mathcal{A}$ if and only if $\mathcal{A}$ has a subalgebra isomorphic to $\mathrm{E}_{n+1}$. If the solution exists, it is unique: the smallest nonzero element of $\mathrm{七}_{n+1}$.

Corollary 3.4. Let $n \geq 2$. The formula $x \equiv(\neg x)^{n-1}$ defines $1 / n$ in $[0,1]_{\mathrm{L}}$.

Corollary 3.5. Any rational number in the unit interval is definable in $[0,1]_{\mathrm{E}}$.

Proof. In $[0,1]_{\mathrm{E}}$, the formulas $x \equiv \overline{0}$ and $x \equiv \overline{1}$ define rationals 0 and 1 . Moreover $1 / n$ is definable for $n \geq 2$ by a formula in one variable. The two-variable formula $\varphi(x, y)$, defined as $\left(y \equiv(\neg y)^{n-1}\right) \cdot(x \equiv m y)$, defines $m / n$ in the variable $x$.

We now establish that, under suitable axioms, variables can play the role of rational truth constants. Let $Q=\left\{q_{m, n} \mid m, n \in N, m \leq n, n>0\right\}$ be a set of variables. We write $q_{m / n}$ instead of $q_{m, n}$. Define a theory $T_{Q}$ in Eukasiewicz logic $\mathrm{E}$, using (only) the variables $Q$. The theory $T_{Q}$ consists of:

- $\operatorname{axiom} q_{0 / n} \equiv \overline{0}$ for each $n>0$;

- axiom $q_{1 / 1} \equiv \overline{1}$;

- axiom $q_{1 / n} \equiv\left(\neg q_{1 / n}\right)^{n-1}$ for each $n \geq 2$;

- axiom $q_{m / n} \equiv m q_{1 / n}$ for each $m \leq n, n \geq 2$.

It follows from Corollary 3.5 that $T_{Q}$ has exactly one model over $[0,1]_{\mathrm{E}}$, namely, the one where each $q_{m / n}$ is interpreted by $m / n$.

Under the axiom defining $1 / n$, in the language containing the corresponding $q$-variable, the value $m / n$ becomes term definable as $m q_{1 / n}$; we might use this term directly, instead of introducing a new variable. It is only introduced for uniformity of presentation.

Lemma 3.6. (Over Lukasiewicz logic,) $T_{Q}$ proves the following:

- each valid bookkeeping formula in the sense of q-variables; e.g., $q_{m / n} \cdot q_{k / l} \equiv q_{(m / n) \cdot(k / l)}$, and analogously for any other bookkeeping formula usually considered; 
- $q_{m / n} \equiv q_{m^{\prime} / n^{\prime}}$ for $m / n=m^{\prime} / n^{\prime}$;

- $q_{m / n} \equiv \overline{1}$ for $m=n$.

Proof. We show the first statement; the rest is analogous. Consider a slightly stronger statement: let $\varphi$ be a particular bookkeeping statement and let $T_{0} \subseteq T_{Q}$ consist of definitions of only those rationals that are used in $\varphi$ and any auxiliary rationals needed to define them; then $T_{0}$ is finite. We have $T_{0} \models_{[0,1]_{\mathrm{L}}} q_{m / n} \cdot q_{k / l} \equiv q_{(m / n) \cdot(k / l)}$ by definition of the $q$-variables. The provability claim follows from finite strong standard completeness of $\mathrm{t}$.

On an analogous note (and with an analogous proof), one might substitute any other family of terms that have been proved to enable implicit definability of the rationals (with a suitable indexing of variables): they will be provable from our axioms (and vice versa, each of the our axioms will be provable from them) as a consequence of finite strong standard completeness.

The axioms just presented are redundant. It is in fact sufficient to define $1 / n$ for each prime $n$. Then not only $m / n$ becomes term definable for each $m \leq n$, but also, for $n, n^{\prime}$ two primes, $1 /\left(k n n^{\prime}\right)$ is term definable for each $k$, because the subalgebra generated by $1 / n$ and $1 / n^{\prime}$ in $[0,1]_{\mathrm{L}}$ contains the value $1 /\left(k n n^{\prime}\right)$ and the algebra $\mathcal{L}_{k n n^{\prime}}$ generated by it; this entails that all of the elements are term definable (see [7] and [23]).

We define a translation $\star$ of formulas of RPL into formulas of $\mathrm{E}$; note that we assume RPL uses a countably infinite set $X$ of variables (we refer to them as $x$-variables). For each variable $x_{i}$ of RPL, $x_{i}^{\star}$ is $x_{i}$, for each constant (other than $\overline{0}$ and $\overline{1}$ ), $(m / n)^{\star}$ is $q_{m / n}$. Extend $\star$ to formulas as commuting with all connectives. If $T$ is a set of RPL-formulas, then $T^{\star}$ is the set of $\star$-translations thereof. observe that $\star$ is a bijection between rational constants of RPL (different from $\overline{0}$ and $\overline{1}$ ) and $q$-variables of $\mathrm{E}$.

Corollary 3.7. 4 Let $T$ be a theory and $\varphi$ a formula in the language of RPL. We have $T \vdash_{\mathrm{RPL}} \varphi$ if and only if $T^{\star} \cup T_{Q} \vdash_{\mathrm{E}} \varphi^{\star}$.

Proof. $\star$-translations of bookkeeping axioms are provable in $T_{Q}$. On the other hand, the $T_{Q}$-axioms become theorems of RPL under de-starring.

As a special case ( $T$ empty), the theory $T_{Q}$ proves (translations of) all theorems or RPL.

Coming back to bookkeeping formulas, let us see how they might provide an alternative implicit definition of the rationals. We limit the presentation to a sketchy example, leaving the rest to the interested reader. Working in a language with $\cdot, \rightarrow$ and $\overline{0}$ as basic connectives, we shall show how to define rationals with a denominator 3 . The following are some (not all) bookkeeping formulas for variables indexed by such rationals:

$$
\begin{aligned}
& x_{2 / 3} \cdot x_{2 / 3} \equiv x_{1 / 3} \\
& x_{1 / 3} \cdot x_{2 / 3} \equiv x_{0} \\
& x_{1 / 3} \cdot x_{1 / 3} \equiv x_{0} \\
& x_{1 / 3} \rightarrow x_{0} \equiv x_{2 / 3}
\end{aligned}
$$

Under the additional information that $x_{0} \equiv \overline{0}$, the last axiom reads $\neg x_{1 / 3} \equiv x_{2 / 3}$, which, combined with the first one, gives $\left(\neg x_{1 / 3}\right)^{2} \equiv x_{1 / 3}$; the latter implies $v\left(x_{1 / 3}\right)=1 / 3$ in $[0,1]_{\mathrm{E}}$ for

${ }^{4}$ Cf. [12], Lemma 3.3.13(2) 
all models $v$ of the four bookkeeping formulas above, appealing to Corollary 3.4. Observations to be made are (a) the four formulas entail (semantically, and hence also syntactically) an instance of $x \equiv(\neg x)^{n-1}$ for $n=3$, and hence define $1 / 3$ in the variable $x_{1 / 3}$; (b) the second and third axiom are redundant; they follow (semantically, and hence syntactically) from the first and the fourth axiom. Analogously, one might find redundancies in bookkeeping for other denominators, and hence also for combining different prime denominators, for example. We do not go into detail.

Thus, bookkeeping formulas are sufficient (and more than that) for implicit definition of all the rationals in $[0,1]_{\mathrm{L}}$.

\subsection{Deductive Beth property}

To formulate Beth property, we recall the definition of the two properties (of explicit and implicit definability) as usually considered, i.e., referring to variables rather than elements of a specific semantics. Within the scope of this subsection we shall use the notions as given in Definition 3.8.

There are at least two ways to render the property in Eukasiewicz logic (see the discussion in [20]); what is studied here is the deductive version of Beth property.

Definition 3.8. Let $\mathrm{L}$ be a logic and $\varphi(x, \tilde{z})$ a term in the language of $\mathrm{L}$.

- $\varphi$ explicitly defines $x$ in $\mathrm{L}$ if and only if there is a term $\delta(\tilde{z})$ (in the $\tilde{z}$-variables only) such that $\varphi(x, \tilde{z}) \vdash_{\mathrm{L}} x \equiv \delta(\tilde{z})$ for each $x$ and $\tilde{z}$

- $\varphi$ implicitly defines $x$ in $\mathrm{L}$ if and only if $\varphi(x, \tilde{z}), \varphi\left(x^{\prime}, \tilde{z}\right) \vdash_{\mathrm{L}} x \equiv x^{\prime}$ for each $x, x^{\prime}$, and $\tilde{z}$

Our earlier (semantic) definitions of term- and implicit definability in $[0,1]_{\mathrm{E}}$ nod to the definitions (for variables) above. In particular, any equation, or finite system thereof, that has a unique solution in a variable $x$ in $[0,1]_{\mathrm{E}}$, translates in the obvious way into a propositional equivalence (a term) that implicitly defines $x$ in the logic $\mathrm{E}$. This is a consequence of the finite strong standard completeness theorem of $\mathrm{E}$.

Lemma 3.9. The term $x \equiv(\neg x)^{n-1}$ implicitly defines $x$ in $\mathrm{E}$.

We say that a logic has the deductive Beth property if, whenever a term $\varphi$ implicitly defines $x$, then the term also explicitly defines $x$.

Corollary 3.10. Eukasiewicz propositional logic does not have the deductive Beth property.

Proof. We have that $x \equiv(\neg x)^{n-1}$ implicitly defines $x$. In order for this formula to explicitly define $x$, an MV-term needs to exist that is provably equivalent to $x$ under $x \equiv(\neg x)^{n-1}$; so in particular, in $[0,1]_{\mathrm{E}}$, the term would have to take the constant value $1 / n$. There is no such MV-term; therefore, $x \equiv(\neg x)^{n-1}$ does not explicitly define $x$ in $\mathrm{E}$.

This result for Eukasiewicz logic was given in [16], addressing the topic in a comprehensive way over a large landscape of logics. See [20] for the more specific area of logics extending Hájek's Basic Fuzzy Logic BL. 


\subsection{Defining the irrationals}

Lemma 3.11. An irrational number $a \in[0,1]$ is not definable by an $M V$-term in $[0,1]_{\mathrm{E}}$.

Proof. If an MV-term $\varphi\left(x_{1}, \ldots, x_{n}\right)$ is satisfiable in $[0,1]_{\mathrm{E}}$, there is a rational $n$-tuple $\left\langle r_{1}, \ldots, r_{n}\right\rangle$ that satisfies $\varphi$. Let $a$ be an irrational. If $\varphi\left(x_{1}, \ldots, x_{n}\right)$ is satisfiable in $[0,1]_{\mathrm{E}}$ and $1 \leq i \leq n$, then there is an evaluation $v$ in $[0,1]_{\mathrm{E}}$ s.t. $v(\varphi)=1$ and $v\left(x_{i}\right) \neq a$.

Nevertheless, if one compromises on the requirement that implicit definitions are finite objects (i.e., given by a term) and if one shifts from finitary to infinitary Łukasiewicz logic $\mathrm{E}_{\infty}$, one can implicitly define each irrational, as we now show.

Our simple construction illustrates the technique, working with only two values. We leave any further elaboration to the reader. We do not discuss or advocate here the necessity of introducing all irrational values as constants.

We shall extend the theory $T_{Q}$. Consider irrationals $a, b \in[0,1]$ s.t. $a \cdot a=b$. Irrationals are cuts on the rationals, and the latter have already been defined by the axioms of $T_{Q}$.

Let

$$
\begin{aligned}
& A_{1}=\{q \in \mathbb{Q} \cap[0,1] \mid q<a\} \\
& A_{2}=\{q \in \mathbb{Q} \cap[0,1] \mid a<q\}
\end{aligned}
$$

and analogously $B_{1}, B_{2}$ for b. Introduce fresh variables (i.e., not occurring in $\left.T_{Q}\right) i_{a}, i_{b}$. Let

$$
T_{Q, a}=T_{Q} \cup\left\{q_{m / n} \rightarrow i_{a} \mid m / n \in A_{1}\right\} \cup\left\{i_{a} \rightarrow q_{m / n} \mid m / n \in A_{2}\right\}
$$

Analogously, define $T_{Q, b}$ for $b$.

Lemma 3.12. In any standard model of $T_{Q, a}$, the variable $i_{a}$ has the value $a$.

Proof. Since $T_{Q}$ only admits canonical interpretations of the $q$-variables in the standard MValgebra, the cut $\left(A_{1}, A_{2}\right)$, captured by the axioms of $T_{Q, a}$, determines the valuation of $i_{a}$.

Let $T_{Q, a, b}$ be the theory whose axioms are the union of axioms for $T_{Q, a}$ and $T_{Q, b}$.

Corollary 3.13. $T_{Q, a, b} \vdash_{\mathrm{E}_{\infty}} i_{a} \cdot i_{a} \equiv i_{b}$.

Proof. Since the values of $i_{a}$ and $i_{b}$ are fixed in $[0,1]_{\mathrm{E}}$ by $T_{Q, a}$ and $T_{Q, b}$, we have $T_{Q, a, b} \models_{[0,1]_{\mathrm{L}}} i_{a} \cdot i_{a} \equiv i_{b}$. Then

$T_{Q, a, b} \vdash_{\mathrm{E}_{\infty}} i_{a} \cdot i_{a} \equiv i_{b}$ by strong standard completeness of $\mathrm{E}_{\infty}$.

As in the case of constants for rationals, one can introduce irrational constants incrementally (as above). It is known that, if $a$ is an irrational in $[0,1]_{\mathrm{L}}$, then the subalgebra generated by $a$ is dense ([9]); as in the case of rationals, when just one value is defined by suitable axioms, other values become term definable within such a theory with an expanded language. 


\subsection{Completeness theorems}

Finally, let us remark on completeness theorems in this new setting. There is nothing to add for general or standard finite strong completeness. However, one might wonder how to spell Pavelka completeness result. Let $T$ be a set of formulas and $\varphi$ a formula of $€$ (possibly with some $q$-variables). We define

$$
\|\varphi\|_{T \cup T_{Q}}=\inf \left\{v(\varphi) \mid v \text { model of } T \cup T_{Q}\right\}
$$

and 5

$$
|\varphi|_{T \cup T_{Q}}=\sup \left\{m / n \mid T \cup T_{Q} \vdash_{\llcorner} q_{m / n} \rightarrow \varphi\right\}
$$

The definition rests on a very convenient indexing of the $q$-variables. The same convention occurs in the usual spelling of the provability degree in RPL: in particular, one can perform computations and take suprema on the names of the constants. Both definition rest on the link between the name of the constant and its intended value; usually the language is chosen in such a way that this link is immediate.

\section{Recognition and complexity}

To provide a sample application of the interpretation result, let us look at the complexity of provability from a finite theory in propositional RPL; in view of the finite strong standard (canonical) completeness theorem for RPL, this is just the finite consequence relation in $[0,1]_{\mathrm{E}}$ (for the propositional language with rational constants). Recall the problem: given a finite theory $T$ and a formula $\varphi$ of RPL, does $T \vdash_{\mathrm{RPL}} \varphi$ ? For an empty $T$, this problem is just theoremhood in RPL. Without wishing to discuss implementation details, we remark that each rational constant is represented as a pair of natural numbers given in binary.

A dual form of the conservativity result gives that provability without constants is a language fragment of provability with constants; for finite theories, the former is coNP-complete, so the latter is coNP-hard. In [13, Hájek argues coNP-containment for (theoremhood and) provability from finite theories in RPL by looking at the standard semantics and asking the reader to verify that the decision method used in [12] for Lukasiewicz logic, which is obtained as a polynomial-time reduction to mixed integer programming, works even under the presence of rational constants in the formulas. Of course, the extension with rational constants is quite natural for the mixed integer programming problem.

It is also unnecessary. The statement of Corollary 3.7 provides a method to argue the result without re-examining the reduction to mixed integer programming: one simply uses the $\star$-translation and appeals to a finite subtheory of $T_{Q}$ to provide a canonical interpretation for the new variables; in particular, for each constant $m / n$, it is necessary to include the defining axiom for $q_{1 / n}$ and $q_{m / n}$ in the finite subtheory. Next to that, the only complexity result needed is that of coNP-completeness of finite consequence relation in $\mathrm{E}$.

A small glitch is that the interpretation provided by $\star$ and (the finite subtheory of) $T_{Q}$ cannot be used in the form given above because they do not yield a polynomial translation: for a given $n$, the innocent-looking abbreviation $\varphi^{n-1}$ in fact stands for a term of exponential size in $|n|$ (for $n$ represented in binary). This problem can be rectified in the usual manner, using new variables for intermediate powers; in the lemma below, powers of MV-terms and

\footnotetext{
${ }^{5}$ Cf. also [14, Theorem 3.1 and above.
} 
the product of $\mathrm{MV}$-terms denoted by $\Pi$ pertain to the multiplication symbol $\cdot$ of the language of MV-algebras.

Lemma 4.1. For $n \in N, n \geq 2$, take the binary representation of $n-1$, i.e., let $n-1=$ $\sum_{i=0}^{m} p_{i} 2^{i}$ with $p_{i} \in\{0,1\}$ and $p_{m}=1$. Let $I=\left\{i \mid p_{i}=1\right\}$. In $[0,1]_{\mathrm{E}}$, the system of equations

$$
\begin{aligned}
y_{0} & =\neg x \\
y_{1} & =y_{0}^{2} \\
y_{2} & =y_{1}^{2} \\
& \vdots \\
y_{m} & =y_{m-1}^{2} \\
x & =\Pi_{i \in I} y_{i}
\end{aligned}
$$

has a unique solution, assigning the value $1 / n$ to $x$.

Proof. The system implies $x=(\neg x)^{n-1}$. This equation has a unique solution $x=1 / n$ in $[0,1]_{\mathrm{E}}([15]$, Lemma 6.3$)$. On the other hand, the assignment $x=1 / n$ determines the values assigned to all the $y$-variables.

In what follows, the $y$-variables from the above lemma are referred to as "auxiliary variables".

Lemma 4.2. 1. For $n, m, I$ as above, the system of formulas

$$
\left\{y_{0} \equiv \neg q_{1 / n}, \bigwedge_{i=1}^{m}\left(y_{i}=y_{i-1}^{2}\right), q_{1 / n}=\Pi_{i \in I} y_{i}\right\}
$$

defines $1 / n$ in the variable $q_{1 / n}$ in $[0,1]_{\mathrm{E}}$.

2. The size of the system is polynomial in the size of $n$.

3. Using an analogous argument, one can polynomially define $m / n$.

Theorem 4.3. Provability from finite theories in RPL is polynomially reducible to provability from finite theories in $\mathrm{E}$.

Proof. Let a finite consecution $(T, \varphi)$ in the language of RPL be given. Let $\left\{m_{1} / n_{1}, \ldots m_{k} / n_{k}\right\}$ be all the rational constants in $T, q$, and let all variables therein be among $\left\{x_{1}, \ldots, x_{l}\right\}$. For $i=1, \ldots, k$ let $T_{i}$ be the set of formulas from Lemma 4.2 (1) and (3), using a fresh pool of auxiliary variables to define each of $1 / n_{i}$ and $m_{i} / n_{i}$. Let $T_{Q}^{f i n}=\bigcup_{i=1}^{k} T_{i}$, and let $T^{\star}$ and $\varphi^{\star}$ result from $T$ and $\varphi$ by replacing its constants with the respective $q$-variables. Then $T \vdash_{\text {RPL }} \varphi$ if and only if $T^{\star} \cup T_{Q}^{\text {fin }} \vdash_{\mathrm{L}} \varphi^{\star}$ as in Theorem 3.7, moreover, the size of $T_{Q}^{\text {fin }}$ (and also $T^{\star}$ and $\left.\varphi^{\star}\right)$ is polynomial in $|T|+|\varphi|$.

Corollary 4.4. Provability from finite theories in RPL is in coNP.

The construction of the polynomial translation is not less laborious than the inclusion of the rational constants in the mixed integer programming problem; one cannot argue that the (coNP-containment) result became simpler by omitting the constants.

However, what the construction shows is that problems such as the computational complexity of RPL are in fact problems about L (using the translation). In other words, there was no question one could ask about complexity of RPL in the first place, other than those already settled by complexity results for $\mathrm{E}$. 
Corollary 3.5 provides a way of defining each of the rationals in Eukasiewicz logic in the sense of Definition 3.2. This is just one among many variants of the implicit definability result. One can observe that the construction rests on a parametrized formula where by varying the parameter one easily gets the definition of the intended constant for each $m / n$.

On the other hand, consider a randomly chosen formula. What, if anything, does it define? We formulate this question as a decision problem, and show that it is hard.

Problem 4.5. Given an $M V$-term of $n$ variables $\varphi\left(x_{1}, \ldots, x_{n}\right)$, an integer $1 \leq i \leq n$, and a rational number $a$, determine whether $\varphi$ defines a in $x_{i}\left(\right.$ in $\left.[0,1]_{\mathrm{E}}\right)$.

The decision problem on the domain of triples $\langle\varphi, i, a\rangle$ as above, consisting of exactly those where $\varphi$ defines $a$ in $x_{i}$ in the standard MV-algebra, is referred to as the DEF problem.

We seek to estimate the complexity of the problem. The estimate will in part be an artefact of our definition. Namely, we have stipulated that each of our defining formulas be satisfiable (in $[0,1]_{\mathrm{E}}$ ); otherwise, if only the second item in Definition 3.2 were used, each unsatisfiable formula would implicitly define every rational (and irrational) in a trivial way. The following lemma reflects our choice.

Lemma 4.6. DEF is NP-hard.

Proof. We reduce SAT to DEF (both notions relate to $[0,1]_{\mathrm{E}}$ ).

The reduction function $f$ assigns, to a given formula $\varphi\left(x_{1}, \ldots, x_{n}\right)$, the triple

$$
\left\langle\varphi \cdot\left(x_{n+1} \equiv \neg x_{n+1}\right), n+1,1 / 2\right\rangle
$$

We show that $\varphi \in$ SAT if and only if $f(\varphi) \in$ DEF.

Let $\varphi$ be satisfiable; then so is $\varphi \cdot\left(x_{n+1} \equiv \neg x_{n+1}\right)$, and moreover, if the latter is satisfied by a $v$, then $v\left(x_{n+1}\right)=1 / 2$, because $1 / 2$ is the only solution to the equation $x=\neg x$ (note $x_{n+1}$ does not occur in $\varphi$ ).

On the other hand, if $\varphi$ is unsatisfiable, then so is $\varphi \cdot\left(x_{n+1} \equiv \neg x_{n+1}\right)$.

Theorem 4.7. DEF is coNP-hard.

Proof. For a given $\langle\varphi, i, a\rangle$ as above, let us consider the condition

$$
\exists v_{[0,1]_{\mathrm{E}}}\left(v(\varphi)=1 \& v\left(x_{i}\right) \neq a\right)
$$

This condition negates the second condition in Definition 3.2. It is in NP (because of small witnesses); to show it is NP-hard, it is enough to consider a variant of the reduction from Lemma 4.6. to find out whether $\varphi\left(x_{1}, \ldots, x_{n}\right) \in \mathrm{SAT}$, ask the algorithm for $\mathrm{D}$ about $\left\langle\varphi \cdot\left(x_{n+1} \equiv \neg x_{n+1}\right), n+1,1 / 4\right\rangle$. If $\varphi$ is satisfiable, then D is satisfied on $f(\varphi)$; if not, then neither is $\varphi \cdot\left(x_{n+1} \equiv \neg x_{n+1}\right)$, so $\mathrm{D}$ fails. So D is NP-complete.

Now we reduce $\bar{D}$ (the complement of $\mathrm{D}$ ), i.e., the set $\langle\varphi, i, a\rangle$ s.t. $v(\varphi)=1$ implies $v\left(x_{i}\right)=a$, to DEF (i.e., to $\bar{D}$ with the additional condition of satisfiability for $\varphi$ ).

Let $\langle\varphi(\tilde{x}), i, a\rangle$ be an instance. Let $\psi\left(x_{i}, \tilde{z}\right)$ be a formula defining $a$ in $x_{i}$ (as earlier in this paper). Note that

(i) $\psi$ is satisfiable, and for any satisfying evaluation $v$, we have $v\left(x_{i}\right)=a$;

(ii) $\psi$ can be chosen so that its size is polynomial in the size of the given instance.

Let $f(\langle\varphi, i, a\rangle)=\langle(\varphi \vee \psi)(\tilde{x}, \tilde{z}), i, a\rangle$. We claim that $\langle\varphi, i, a\rangle$ satisfies (D) if and only if $f(\langle\varphi, i, a\rangle) \in \mathrm{DEF}$. 
If $\langle\varphi, i, a\rangle$ satisfies $\bar{D}$, then $\varphi \vee \psi$ is satisfiable (because $\psi$ is), and satisfies the condition of $\bar{D}$, because both $\varphi$ and $\psi$ satisfy it. So $f(\langle\varphi, i, a\rangle) \in$ DEF.

If $\langle\varphi, i, a\rangle$ satisfies (D), then there is a $v$ s.t. $v(\varphi)=1$ and $v\left(x_{i}\right) \neq a$. This is also true about $\varphi \vee \psi$.

(The proof of) Theorem 4.7 answers the complexity question for a modification of Definition 3.2 omitting the satisfiability requirement. Such a problem is coNP-complete.

\section{More on RPL and $\mathbf{L}$}

As stated already, $\mathrm{E}$ is a syntactic fragment of RPL: well-formed formulas of $\mathrm{E}$ are exactly those well formed formulas of RPL that do not contain any constants other than $\overline{0}$ and $\overline{1}$. Dually, RPL is a conservative extension of .

The conservative extension statement, however, does not quite capture the tight relation between RPL and $\mathrm{E} 6$ To appreciate that, consider for example that Peano arithmetic is a conservative extension of Presburger arithmetic. But Peano arithmetic does not stand to Presburger arithmetic as RPL stands to $\mathrm{E}$.

Rather, what we have in RPL is an axiomatic expansion of the language as exemplified by extending the theory of groups, presented with the binary group operation only, with a new constant for the neutral element. This is an extension by definition (so it is conservative) and because we can prove existence and unicity of the solution to $x \circ x=x$, the new constant for the neutral element can be eliminated. In the case of RPL, [14, Theorem 2.6] gives the exact sense in which constants can be eliminated, as mentioned in Section 1 above.

Not so much the conservativity result, but predominantly the faithful interpretation result suggests that the logics stand too close to each other to be even considered as two genuine systems, except for some very theoretical considerations (such as the set of term-definable functions in the standard semantics, for example); for practical applications, however, it is a matter of convenience which of the two logics is used.

\section{On precision and vagueness}

Within the framework of Eukasiewicz logic, with or without constants in the language, one can be surgically precise about truth values ascribed to particular propositions. We have seen that, under a simple theory such as $T_{Q}$, one can pinpoint the truth value of a proposition $p$ to any rational number by positing $p \equiv q_{m / n}$. There is no talk about valuations here: $T_{Q}$ and $p \equiv q_{m / n}$ MV-terms or sets thereof 7 Given that the rationals are dense within [0,1], this level of precision seems sufficient for practical purposes, which presuppose finiteness. Should irrational values be needed, however (thus forfeiting finiteness), we have shown how to obtain them switching the underlying logic to the infinitary logic $\mathrm{E}_{\infty}$.

In recent papers concerning the modelling of vague predicates with fuzzy logic, the predicate tall is considered as an example amenable to modelling by the apparatus of formal fuzzy

\footnotetext{
${ }^{6}$ This is also reflected in [14, §3]: "Lemma 2.3 shows that RPL $\forall$ is a very conservative extension indeed of $\mathrm{\forall}$. There is a sense in which even its new formulae don't express anything which can't be expressed by old formulae."

${ }^{7}$ Admittedly, $T_{Q}$ imposes its own semantics: it does not have a model over algebras that do not contain a copy of the rationals.
} 
logic. It is so amenable because it is a vague way of referring to an easily measurable quantity, namely height, which is not vague, but is measured on a linear scale.

The claim here is not that Eukasiewicz logic or its unspecified theories prove $p$ to have the truth value $m / n$ or any other value for that matter (unless $p$ turns out to be a theorem or a contradiction of Łukasiewicz logic, of course). Specific theories may indeed prove $p \equiv q_{m / n}$, but then (trivially) the axioms of such theories need to be at least as strong as $p \equiv q_{m / n}$. Łukasiewicz logic however, as a formal system, does not commit to such ad hoc stipulations, but merely makes it possible to express them, and to enable deduction on them.

One possible rephrasing of the above is that, right side of the turnstile, we know rather little of how our "truth constants" came to be; bar some notational conventions (helping us to distinguish between constants and variables), we are presented with a set of syntactic objects that obey some bookkeeping statements.

Detailed accounts [18] and [1], echoing also earlier works of Hájek, explain that (Eukasiewicz or other) fuzzy logic does not insist on assigning specific real values to propositions, but rather, broadly speaking, studies consequence on, and reasoning with, propositions that in principle admit these truth values. These accounts are offered in response to some attempts at refutation of fuzzy logic on the ground of the artificial precision problem that points out the lack of incentives for preferring one intermediate truth value over another one for particular propositions.

We have argued that precision can be neatly captured by the syntax of Eukasiewicz logic. All of that is happening left of the turnstile. We have also reminded ourselves of the simple fact that it is nontrivial to recognize what precisely an arbitrary theory implicitly defines. One might, therefore, understand the account presented here as a supportive argument for the thesis that precision happens in fuzzy logic, and if it happens, it must happen within the assumptions before it can happen within the conclusions, which seems to be one of the main points of the above papers.

\section{Conclusion}

Thanks to finite strong standard completeness of Łukasiewicz logic, our semantic rendering of implicit definability blends naturally with the more commonly given implicit definability notions that refer to variables (as when introducing Beth property, see [16, 20]) or connectives (as in [2, 3]), i.e., syntactic objects.

The findings of Section 3 and 4 seem to confirm the ambivalent status of constants in Eukasiewicz logic: they are useful but dispensable abbreviations, with statements of Łukasiewicz logic with constants being expressible in Łukasiewicz logic without any constants but those that are inherent to it. It may well be that keeping the constants is "more elegant", as asserted by Hájek in [12], or that "... even for partial truth, Rational Pavelka logic deals with exactly the same logic as Eukasiewicz logic - but in a very much more convenient way", as 14 remarks. We do not attempt to argue for or against these views. What is argued here, however, is that metamathematical statements about Rational Pavelka logic naturally translate to statements about (certain theories over) Eukasiewicz logic without any added constants. In particular, it is not the case that Eukasiewicz logic with truth constants is richer, more expressive, or more general than Łukasiewicz logic without constants. 
Acknowledgements. The author was supported by CE-ITI and GAČR under the grant number GBP202/12/G061, and by the long-term strategic development financing of the Institute of Computer Science RVO:67985807.

\section{References}

[1] Libor Běhounek. In Which Sense Is Fuzzy Logic a Logic for Vagueness? In Thomas Łukasiewicz, Rafael Peñaloza, and Anni-Yasmin Turhan, editors, Proceedings of the First Workshop on Logics for Reasoning about Preferences, Uncertainty, and Vagueness, pages 26-39, Vienna, 2014.

[2] Xavier Caicedo. Implicit connectives of algebraizable logics. Studia Logica, 78:155-170, 2004.

[3] Xavier Caicedo. Implicit operations in MV-algebras and the connectives of Eukasiewicz logic. In Algebraic and Proof-Theoretic Aspects of Many-Valued Logics, volume 4460 of Lecture Notes in Computer Science, pages 50-68. Springer, 2007.

[4] Roberto Cignoli, Itala M.L. D’Ottaviano, and Daniele Mundici. Algebraic Foundations of Many-Valued Reasoning, volume 7 of Trends in Logic. Kluwer, Dordrecht, 1999.

[5] Petr Cintula. A note on axiomatizations of Pavelka-style complete fuzzy logics. Fuzzy Sets and Systems, 292:160-174, 2016.

[6] Petr Cintula, Francesc Esteva, Joan Gispert, Lluís Godo, Franco Montagna, and Carles Noguera. Distinguished algebraic semantics for t-norm based fuzzy logics: Methods and algebraic equivalencies. Annals of Pure and Applied Logic, 160(1):53-81, 2009.

[7] Antonio Di Nola and Ioana Leuştean. Łukasiewicz Logic and MV-Algebras. In Petr Cintula, Petr Hájek, and Carles Noguera, editors, Handbook of Mathematical Fuzzy Logic, volume 2, pages 469-583. College Publications, 2011.

[8] Francesc Esteva, Joan Gispert, Lluís Godo, and Carles Noguera. Adding truth-constants to logics of continuous t-norms: Axiomatization and completeness results. Fuzzy Sets and Systems, 158(6):597-618, 2007.

[9] Hernando Gaitán. The number of simple one-generated bounded commutative BCKchains. Mathematica Japonica, 38(3):483-486, 1993.

[10] Joan Gispert. Universal Classes of MV-chains with Applications to Many-valued Logic. Mathematical Logic Quarterly, 42:581-601, 2002.

[11] Joseph Amadee Goguen. The logic of inexact concepts. Synthese, 19(3-4):325-373, 1969.

[12] Petr Hájek. Metamathematics of Fuzzy Logic, volume 4 of Trends in Logic. Kluwer, Dordrecht, 1998.

[13] Petr Hájek. Computational complexity of t-norm based propositional fuzzy logics with rational truth constants. Fuzzy Sets and Systems, 157(5):677-682, 2006. 
[14] Petr Hájek, Jeff Paris, and John C. Shepherdson. Rational Pavelka logic is a conservative extension of Łukasiewicz logic. Journal of Symbolic Logic, 65(2):669-682, 2000.

[15] Zuzana Haniková and Petr Savický. Term satisfiability in $\mathrm{FL}_{e w}$-algebras. Theoretical Computer Science, 631:1-15, 2016.

[16] Eva Hoogland. Definability and Interpolation: Model-theoretic investigations. PhD thesis, Institute for Logic, Language and Computation, 2001.

[17] Jan Łukasiewicz and Alfred Tarski. Untersuchungen über den Aussagenkalkül. Comptes Rendus des Séances de la Société des Sciences et des Lettres de Varsovie, cl. III, 23(iii):30-50, 1930.

[18] Vincenzo Marra. The Problem of Artificial Precision in Theories of Vagueness: A Note on the Rôle of Maximal Consistency. Erkenntnis, 79(5):1015-1026, 2014.

[19] Robert McNaughton. A theorem about infinite-valued sentential logic. Journal of Symbolic Logic, 16(1):1-13, 1951.

[20] Franco Montagna. Interpolation and Beth's property in propositional many-valued logics: A semantic investigation. Annals of Pure and Applied Logic, 141(1-2):148-179, 2006.

[21] Daniele Mundici. Advanced Eukasiewicz Calculus and MV-Algebras, volume 35 of Trends in Logics. Springer, 2011.

[22] Jan Pavelka. On fuzzy logic I, II, III. Zeitschrift für Mathematische Logik und Grundlagen der Mathematik, 25:45-52, 119-134, 447-464, 1979.

[23] Antoni Torrens. Cyclic elements in MV-algebras and Post algebras. Mathematical Logic Quarterly, 40(4):431-444, 1994. 